\title{
VARIAÇÃO DAS CONDIÇÕES DE SINTERIZAÇÃO E SEUS EFEITOS NO SISTEMA Al ${ }_{2} \mathrm{O}_{3}-\mathrm{Nb}_{2} \mathrm{O}_{5}-\mathrm{LiF}^{*}$
}

\author{
Jheison Lopes dos Santos ${ }^{1}$ \\ Alaelson Vieira Gomes ${ }^{2}$ \\ Rubens Lincoln Santana Blazutti Marçal ${ }^{3}$ \\ Daniel Navarro da Rocha ${ }^{4}$ \\ Édio Peireira Lima Jr. ${ }^{5}$
}

\section{Resumo}

O presente estudo teve por objetivo principal produzir um cerâmico composto de alumina, nióbia e fluoreto de lítio de forma a otimizar o uso do forno na etapa de sinterização. Para isso, ponderou-se a respeito da necessidade ou não de utilização de um patamar a $1000^{\circ} \mathrm{C}$ utilizado em estudos anteriores. Além disso, buscou-se diminuir a temperatura de sinterização, assim como o tempo de permanência em tais temperaturas. O composto cerâmico $\left(\mathrm{Al}_{2} \mathrm{O}_{3}-4 \% \mathrm{Nb}_{2} \mathrm{O}_{5}\right)-0,5 \% \mathrm{LiF}$ foi sinterizado a três temperaturas diferentes $(1200,1250$ e $1300 \stackrel{\circ}{\circ}$ ), com tempo de patamar de $1 \mathrm{~h}$. Foi realizado ensaio de Arquimedes para analisar sua densificação e determinar sua densidade. As fases formadas nessas temperaturas foram identificadas por difração de raios- $X$. Os resultados mostraram que a maior densificação foi obtida para a maior temperatura, de $1300 \stackrel{\circ}{\circ}$. Foi possível observar também que um patamar de apenas $1 \mathrm{~h}$ na temperatura de sinterização de $1300{ }^{\circ} \mathrm{C}$ apresentou igual densificação a de outros trabalhos, com tempo de patamar de $3 \mathrm{~h}$. Este fato sugere que a temperatura tem maior influência na sinterização do que o tempo de permanência. Verificou-se ainda que as mesmas fases foram formadas nas três temperaturas investigadas, sendo tais fases as mesmas detectadas em trabalhos anteriores com patamares maiores. Isso mostra que o prolongado tempo de $3 \mathrm{~h}$ no patamar de sinterização apenas favorece o crescimento dos grãos, bastando apenas a permanência de $1 \mathrm{~h}$ para alcançar os resultados desejados e com grãos menores.

Palavras-chave: $\mathrm{Al}_{2} \mathrm{O}_{3}$; Densificação; Processo de sinterização.

\section{VARIATION OF SINTERIZATION CONDITIONS AND ITS EFFECTS ON THE $\mathrm{Al}_{2} \mathrm{O}_{3}-\mathrm{Nb}_{2} \mathrm{O}_{5}-\mathrm{LiF}$ SYSTEM}

\section{Abstract}

The main objective of this study was to produce a ceramic composed of alumina, niobia and lithium fluoride in order to optimize the use of the furnace in the sintering stage. For this, the necessity or not of using a plateau at $1000^{\circ} \mathrm{C}$ used in previous studies was pondered. In addition, it was sought to reduce the sintering temperature, as well as the permanence time at such temperatures. The ceramic compound $\left(\mathrm{Al}_{2} \mathrm{O}_{3}-4 \% \mathrm{Nb}_{2} \mathrm{O}_{5}\right)-0.5 \% \mathrm{LiF}$ was sintered at three different temperatures $(1200,1250$ and $1300{ }^{\circ} \mathrm{C}$ ), with a threshold time of $1 \mathrm{~h}$. Archimedes test was performed to analyze its densification and determine its density. The phases formed at these temperatures were identified by X-ray diffraction. The results showed that the higher densification was obtained for the higher temperature of $1300 \stackrel{\circ}{ } \mathrm{C}$. It was also possible to observe that a plateau of only $1 \mathrm{~h}$ in the sintering temperature of $1300^{\circ} \mathrm{C}$ presented the same densification as that of other works, with a plateau time of $3 \mathrm{~h}$. This fact suggests that the temperature has a greater influence on the sintering than the plateau time. It was also verified that the same phases were formed in the three investigated temperatures, such phases being the same ones detected in previous works with longer plateaus. This shows that the prolonged time of $3 \mathrm{~h}$ at the sintering 
temperature only favors the growth of the grains, with only $1 \mathrm{~h}$ remaining to achieve the desired results and with smaller grains.

Keywords: $\mathrm{Al}_{2} \mathrm{O}_{3}$; Densification; Sintering process

1 Doutor em Ciência dos Materiais, Pós-Doutorando, Seção de Engenharia Mecânica e de Materiais, Instituto Militar de Engenharia, Rio de Janeiro, RJ, Brasil.

2 Doutor em Ciência dos Materiais, Professor, Seção de Engenharia Mecânica e de Materiais, Instituto Militar de Engenharia, Rio de Janeiro, RJ, Brasil.

3 Doutor em Ciência dos Materiais, Pós-Doutorando, Seção de Engenharia Mecânica e de Materiais, Instituto Militar de Engenharia, Rio de Janeiro, RJ, Brasil.

4 Doutor em Ciência dos Materiais, Pós-Doutorando, Seção de Engenharia Mecânica e de Materiais, Instituto Militar de Engenharia, Rio de Janeiro, RJ, Brasil.

5 Mestre em Ciência dos Materiais, Professor, Seção de Engenharia Mecânica e de Materiais, Instituto Militar de Engenharia, Rio de Janeiro, RJ, Brasil. 


\section{INTRODUÇÃO}

O uso de aditivos em frações relativamente pequenas é de grande importância no aumento de suas propriedades, aliado ao controle microestrutural. No grupo das principais cerâmicas estudadas, estão a zircônia, a ítria, o carbeto de silício, 0 carbeto de boro, e a alumina [1-6]. A alumina $\left(\mathrm{Al}_{2} \mathrm{O}_{3}\right)$, mesmo como um dos mais importantes cerâmicos de engenharia, apresenta características negativas, como baixa resistência à tração, alta fragilidade e baixa tenacidade [7-10]. Para sanar suas desvantagens, vários aditivos são utilizados como agentes que otimizam a sinterização. Dentre os aditivos mais utilizados, está o óxido de nióbio $\left(\mathrm{Nb}_{2} \mathrm{O}_{5}\right)$ [1113], principalmente pelo seu potencial em reduzir a temperatura de sinterização da alumina para $1450{ }^{\circ} \mathrm{C}$ com a adição em peso de $4 \%[14,15]$.

Outro aditivo utilizado em cerâmicos à base de $\mathrm{Al}_{2} \mathrm{O}_{3}$ é o fluoreto de lítio (LiF), por contribuir na formação de fases que sofram fusão durante a sinterização, otimizando o processo [16-18]. Santos [18] otimizou a fração de 0,5\% de LiF ao sistema Al2O3$4 \% \mathrm{Nb} 2 \mathrm{O} 5$ como a adição adequada para promover superior densificação e propriedades satisfatórias. Para a sinterização a alumina, costuma-se utilizar um patamar de sinterização de $3 \mathrm{~h}$.

Como a sinterização é um processo termicamente ativado, busca-se otimizar os fatores tempo e temperatura, a fim de se obter resultados satisfatórios de densificação e propriedades. Dessa forma, esse trabalho teve por objetivo analisar a consequência da redução do tempo e da temperatura do patamar de sinterização, e comparar com outras rotas de sinterização utilizada em pesquisas anteriores. As temperaturas utilizadas foram de 1200, 1250 e $1300^{\circ} \mathrm{C}$, com redução na duração do patamar de $3 \mathrm{~h}$ para $1 \mathrm{~h}$, além da eliminação do patamar de $1000^{\circ} \mathrm{C}$. Dessa forma, a duração do ciclo de sinterização foi reduzida em $3 \mathrm{~h}$.

\section{MATERIAIS E MÉTODOS}

\subsection{Processamento cerâmico}

Foram misturados os pós de alumina (Treibacher Scheifmittel, Brasil), niobia (CBMM, Brasil) e fluoreto de lítio (Vetec, Brasil), de elevada pureza (superiores a 98,5\%), com o ligante orgânico polietileno glicol (PEG) 400 (Vetec), para conferir maior resistência mecânica do corpo cerâmico a verde. A mistura cerâmica precursora é composta por $94,53 \%$ de alumina, 3,94\% de nióbia e 1,53\% do ligante orgânico, pois esta apresentou excelentes propriedades [14,15,18]. Para a pesagem, utilizou-se a balança digital AG 200, da Gehaka, com precisão de 0,1 mg.

Como ligante, foi utilizado o PEG, que foi aquecido até $70^{\circ} \mathrm{C}$ e então dissolvido em água deionizada em um béquer antes de ser adicionado à mistura. Esse procedimento foi realizado para potencializar as propriedades do ligante [14].

Para conferir homogeneização adequada dos pós e do ligante numa moagem em meio líquido, adicionou-se água deionizada. Foi utilizado o moinho MA 500, da marca Marconi, com bolas de alumina com tamanhos variados. O processo de cominuição e homogeneização da mistura teve duração de 8 horas. Utilizou-se jarro do moinho revestido de alumina em seu interior, evitando uma possível adição de novos materiais.

Posteriormente, efetuou-se a secagem do pó úmido em uma estufa da marca ELKA na temperatura de $70^{\circ} \mathrm{C}$ por $48 \mathrm{~h}$. A mistura seca dos pós foi macerada e desaglomerada em gral e pistilo cerâmicos. Após isso, peneirou-se a mistura com 
uma peneira DIN 4188 de abertura de 0,255 mm, com o auxílio de um agitador de peneiras da marca GF modelo TWB. Em seguida à preparação da mistura inicial de referência, adicionou-se $0,5 \%$ em peso de fluoreto de lítio. Para isso, foram reproduzidas as mesmas etapas de processamento realizadas para a obtenção da mistura inicial.

A próxima etapa foi a prensagem uniaxial a frio, onde ocorreu a conformação mecânica dos pós e a produção do corpo verde. Uma matriz de $20 \mathrm{~mm}$ de diâmetro interno foi utilizada para a obtenção dos 15 corpos de prova, cada um com 5 gramas de pó cerâmico, para o estudo do comportamento de sinterização, através das medidas de densidade e do percentual de densificação. Para prensagem das matrizes foi utilizada uma prensa hidráulica com capacidade de 30 toneladas da marca SKAY. Inicialmente, aplicou-se uma pré-carga, de $15 \mathrm{MPa}$, para deixar a camisa flutuante suspensa. Em seguida, aumentou-se gradativamente a carga aplicada, até obter uma pressão total de $50 \mathrm{MPa}$, pressão utilizada em outros trabalhos $[14,18]$ e que apresentou bons resultados, e na região de segurança do valor de pressão otimizado por Trindade [15], de $60 \mathrm{MPa}$, para prevenir introdução de defeitos. A sinterização das amostras foi realizada num modelo de forno FE1700, da marca INTI, com o ciclo térmico adotado na sinterização descrito a seguir:

1) Aquecimento de $25^{\circ} \mathrm{C}$ a $158^{\circ} \mathrm{C}$ à razão de $1^{\circ} \mathrm{C} / \mathrm{min}$;

2) Plantio de aquecimento de $158^{\circ} \mathrm{C}$ durante $1 \mathrm{~h}$;

3) Aquecimento de $158^{\circ} \mathrm{C}$ a $375^{\circ} \mathrm{C}$ à razão de $1^{\circ} \mathrm{C} / \mathrm{min}$;

4) Aquecimento de $375^{\circ} \mathrm{C}$ a $1000^{\circ} \mathrm{C}$ à razão de $8{ }^{\circ} \mathrm{C} / \mathrm{min}$;

5) Aquecimento a partir de $1000^{\circ} \mathrm{C}$ até à temperatura final de sinterização $\left(1200{ }^{\circ} \mathrm{C}\right.$, $1250^{\circ} \mathrm{C}$, ou $1300^{\circ} \mathrm{C}$ ) à razão de $5^{\circ} \mathrm{C} / \mathrm{min}$;

7) Patamar de sinterização à temperatura de sinterização final $\left(1200^{\circ} \mathrm{C}, 1250^{\circ} \mathrm{C}\right.$, ou $1300^{\circ} \mathrm{C}$ ) durante $1 \mathrm{~h}$.

8) Arrefecimento a uma taxa de $3^{\circ} \mathrm{C} / \mathrm{min}$ até $700{ }^{\circ} \mathrm{C}$, quando o forno foi desligado e arrefecido por sua própria inércia.

\subsection{Caracterizações}

\subsubsection{Densidade e Densificação}

A densidade teórica dos corpos sinterizados foi calculada com base na regra das misturas, através dos valores teóricos de densidade conhecidos para a alumina $\left(3,98 \mathrm{~g} / \mathrm{cm}^{3}\right)$, a nióbia $\left(4,60 \mathrm{~g} / \mathrm{cm}^{3}\right)$ e o fluoreto de lítio $\left(2,64 \mathrm{~g} / \mathrm{cm}^{3}\right)$. Assim, foi obtido o valor de $3,998 \mathrm{~g} / \mathrm{cm}^{3}$ para a densidade teórica $\left(\rho_{\text {teo }}\right)$ da composição $99,5 \%$ (96\% $\left.\mathrm{Al}_{2} \mathrm{O}_{3}+4 \% \mathrm{Nb}_{2} \mathrm{O}_{5}\right)+0,50 \% \mathrm{LiF}$.

Após a sinterização, as amostras foram submetidas ao ensaio de Arquimedes, de acordo com a norma ABNT NBR 6220 [19], com o intuito de calcular a densidade aparente e então a densificação com base na densidade teórica.

O ensaio foi realizado fervendo as amostras em água pura por uma hora, com o objetivo de remover o ar e as impurezas dos poros. Em seguida, cada amostra, suspensa por um suporte, foi imersa em água deionizada permitindo aferir a massa imersa $\left(m_{i}\right)$. Então, com o auxílio de um pano úmido para retirar o excesso de água da superfície dos corpos de prova, obteve-se a massa úmida $\left(m_{u}\right)$. Por último, a massa seca $\left(m_{s}\right)$ foi obtida após as amostras permanecerem durante uma hora na estufa de secagem da marca ELKA. 
A densidade aparente $\left(\rho_{a p}\right)$ e a densificação $\left(d e n s_{\%}\right)$ foram calculadas conforme as equações 1 e 2 , em que $\rho_{\text {liq }}$ corresponde à densidade da água utilizada no ensaio, considerada como $1,0 \mathrm{~g} / \mathrm{cm}^{3}$.

$$
\begin{array}{r}
\rho_{a p}=\frac{m_{s}}{m_{u}-m_{i}} \rho_{l i ́ q} \\
\text { densi }_{\%}=\frac{\rho_{a p}}{\rho_{\text {teo }}} \times 100 \%
\end{array}
$$

\subsubsection{Difração de Raios-X}

A análise das fases formadas foi obtida através da técnica de difração de raios-X. Foi realizada à temperatura ambiente em um difratômetro modelo X'Pert Pro, da Panalytical. A operação se deu com uma tensão de $45 \mathrm{kV}$ e corrente elétrica de 40 $\mathrm{mA}$, na faixa $20^{\circ}<2 \theta<80^{\circ}$, com passo de $0,02^{\circ}$ e radiação de $\mathrm{Cu}_{\alpha}$, com tempo de coleta de $2 \mathrm{~s}$.

\section{RESULTADOS E DISCUSSÃO}

\subsection{Densidade e Densificação}

A Tabela 1 apresenta valores de densidade e densificação médias dos três grupos de amostras submetidos a temperaturas de sinterização diferentes. Os valores de densificação representam o percentual da densidade aparente em relação à densidade teórica, calculada pela regra da mistura.

Tabela 1. Propriedades Elásticas da composição otimizada.

\begin{tabular}{lcccc}
\hline Amostras & $\begin{array}{c}\text { Densidade } \\
\left(\mathbf{g} / \mathbf{c m}^{3}\right)\end{array}$ & $\begin{array}{c}\text { Densificação } \\
(\%))\end{array}$ & $\boldsymbol{v}$ & $\begin{array}{c}\lambda \\
(\mathbf{G P a})\end{array}$ \\
\hline $1200{ }^{\circ} \mathrm{C}$ & $232,935 \pm$ & $81,94 \pm 0,26$ & 0,24 & 86,90 \\
& 5,052 & & & $\pm 1,59$ \\
\hline $1250 \stackrel{\circ}{ } \mathrm{C}$ & $265,414 \pm$ & $83,74 \pm 0,11$ & 0,24 & 99,03 \\
& 4,630 & & & $\pm 1,61$ \\
\hline $1300 \stackrel{\circ}{ } \mathrm{C}$ & $289,754 \pm$ & $88,96 \pm 0,34$ & 0,24 & 108,11 \\
& 4,908 & & & $\pm 1,87$ \\
\hline
\end{tabular}

Em trabalhos anteriores [18], a escolha do patamar de 1 hora em $1000^{\circ} \mathrm{C}$ foi feita para garantir a fusão de todo o LiF, que tem ponto de fusão na ordem de $850^{\circ} \mathrm{C}$. Santos [18], utilizando mesma composição e temperatura de sinterização de $1300{ }^{\circ} \mathrm{C}$ com patamar de 3h, também obteve densificação da ordem de $89 \%$. De acordo com os resultados, a retirada desse patamar de $1000{ }^{\circ} \mathrm{C}$ não alterou efetivamente $\mathrm{O}$ resultado da sinterização das amostras, bem como a redução do patamar. Foi verificado que há formação de $\mathrm{LiNbO}_{3}$ em temperaturas inferiores a fusão do $\mathrm{LiF}$ [20] e cujo ponto de fusão é aproximadamente de $1260^{\circ} \mathrm{C}$, justificando a exclusão de tal patamar de temperatura.

Observando o gráfico da Figura 1, é possível notar a evolução da densificação com a temperatura. Cabe ressaltar que o aumento da densificação quando a temperatura de sinterização é alterada de $1250^{\circ} \mathrm{C}$ para $1300^{\circ} \mathrm{C}$ é consideravelmente expressivo se comparado com a alteração de $1200^{\circ} \mathrm{C}$ para $1250^{\circ} \mathrm{C}$. A existência de uma fase de niobato de lítio $\left(\mathrm{LiNbO}_{3}\right)$, detectada por $\mathrm{DRX}$, sugere uma explicação para tal comportamento, pois a fusão dessa fase acontece entre $1250^{\circ} \mathrm{C}$ e $1300^{\circ} \mathrm{C}$. Isso faz 
surgir uma fase líquida durante a sinterização. Essa fase ajuda a aumentar a densificação, porque eleva as taxas de difusão, proporcionando uma sinterização mais rápida e/ou com temperaturas menores [21]. Então, devido à presença de fase líquida no processo de sinterização, que é termicamente ativado, explica-se como foram obtidos os valores de densificação significativos para as amostras sinterizadas a $1300^{\circ} \mathrm{C}$, mesmo diminuindo o último patamar em 2 horas.

Isso sugere que o fator temperatura é mais influente na sinterização do que o fator tempo.

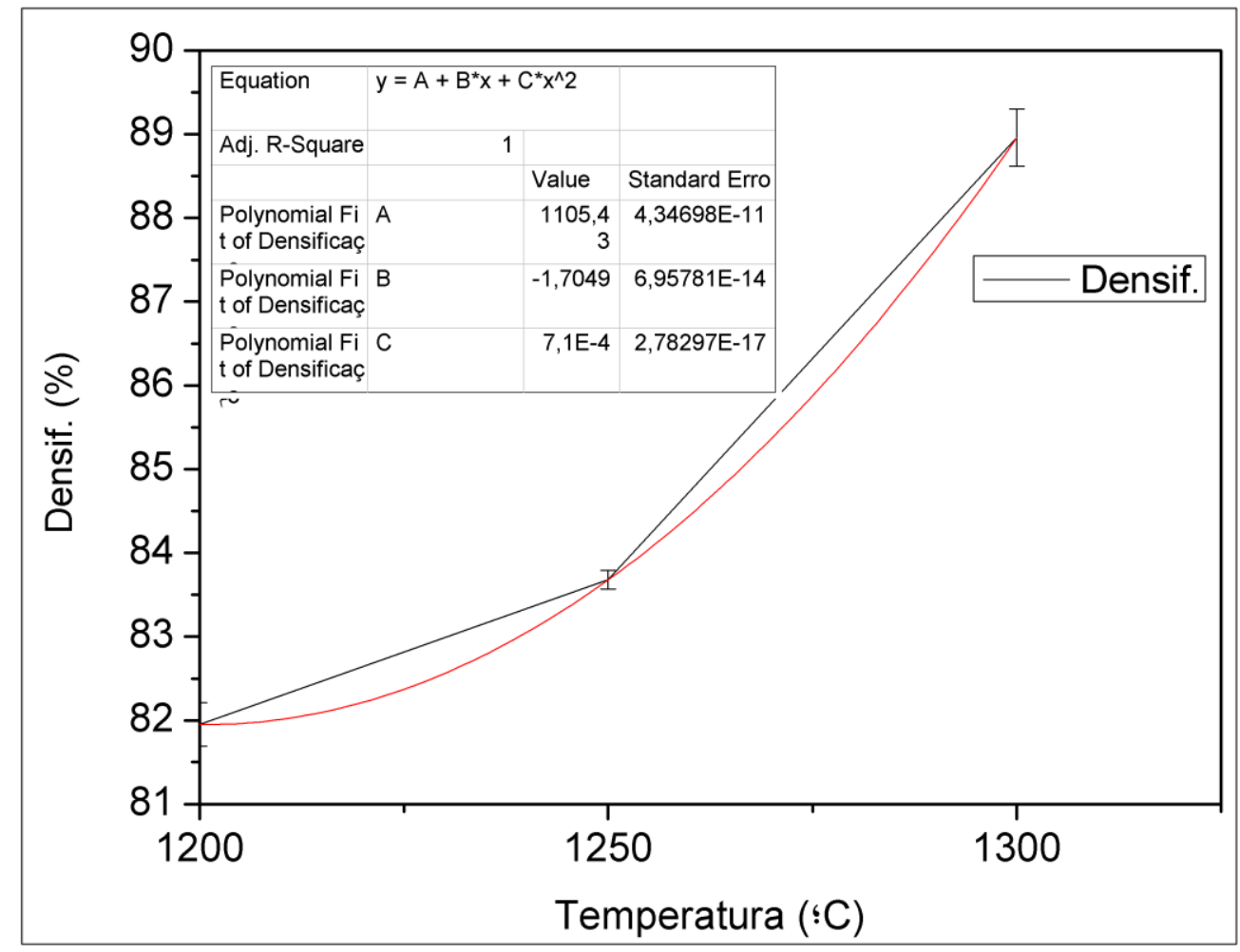

Figura 1. Densificação média das amostras em função da temperatura de sinterização.

\subsection{Difração de Raios-X}

A Figura 2 apresenta os difratogramas obtidos para as amostras sinterizadas nas temperaturas de $1200^{\circ} \mathrm{C}, 1250^{\circ} \mathrm{C}$ e $1300 \stackrel{\circ}{\circ}$. As fases identificadas foram a alumina $\left(\mathrm{Al}_{2} \mathrm{O}_{3}\right.$ ) (JCPDS 00-046-1212), niobato de alumínio (AINbO4) (JCPDS 00-026-0030), $\mathrm{Nb}_{3} \mathrm{O}_{7} \mathrm{~F}$ (JCPDS 00-018-0915), e $\mathrm{LiNbO}_{3}$ (JCPDS 00-020-0631), as mesmas das obtidas por Santos [18]. Os difratogramas apresentaram grande semelhança, com pequenas diferenças apenas nas intensidades de alguns picos. Dessa forma, podese observar que não houve formação de fases distintas para cada uma das temperaturas.

Portanto, as mesmas fases coexistem nas três temperaturas de sinterização investigadas. Isso reforça que o grande benefício na densificação a $1300 \stackrel{\circ}{ } \mathrm{C}$ se deu pela fusão da fase $\mathrm{LiNbO}_{3}$. Aliado a isso, essa temperatura representa uma quantidade maior de energia fornecida ao sistema para promover a sinterização de forma mais satisfatória. Santos sugere que a fase $\mathrm{Nb}_{3} \mathrm{O}_{7} \mathrm{~F}$ possa ter atuado como fase catalisadora para a sinterização. Além disso, a redução na duração do patamar 
influencia no tamanho dos grãos, tendendo a reduzi-lo por não fornecer tempo para promover seus crescimentos.

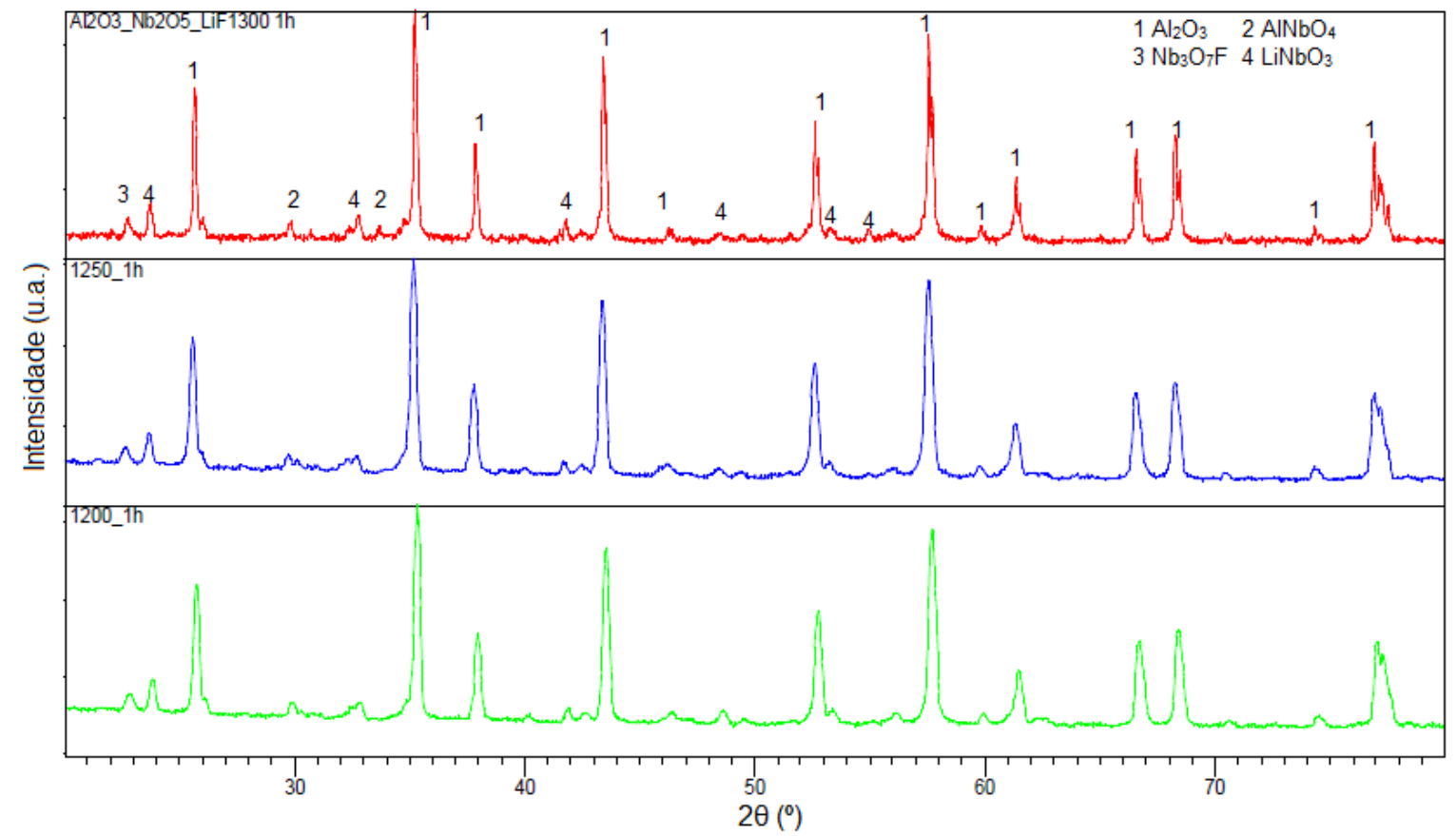

Figura 2. Difratograma das amostras sinterizadas nas diferentes temperatura.

\section{CONCLUSÕES}

Neste trabalho, foi investigada a influência da redução da temperatura de sinterização e de seu patamar no composto cerâmico $\left(\mathrm{Al}_{2} \mathrm{O}_{3}-4 \% \mathrm{Nb}_{2} \mathrm{O}_{5}\right)-0,5 \% \mathrm{LiF}$, a fim de otimizar sua sinterização e baratear ainda mais sua produção. Inicialmente foi investigada sua densificação, relacionando-a com a temperatura de sinterização e fases obtidas. Dessa forma, pode-se concluir que:

1) A eliminação do patamar de $1000^{\circ} \mathrm{C}$ não influenciou na densificação.

2) A redução do patamar de sinterização de $3 \mathrm{~h}$ para $1 \mathrm{~h}$ não afetou negativamente a densificação, ao se comparar com o trabalho realizado por Santos, em se tratando da temperatura de $1300 \stackrel{\circ}{\circ}$.

3) Dessa forma, mostra a redução total em 3h não prejudicou a sinterização do composto cerâmico estudado mediante os valores de densificação obtidos, além de ajudar no controle do crescimento dos grãos.

4) Os resultados de DRX apontaram a formação das mesmas fases nas três temperaturas estudadas, inclusive em comparação com pesquisa anterior [18] utilizando patamares mais longos.

5) A significativa elevação na densificação de $1250^{\circ} \mathrm{C}$ para $1300^{\circ} \mathrm{C}$ foi associada à fusão da fase $\mathrm{LiNbO}_{3}$, promovendo sinterização em presença de fase líquida.

6) Estas significativas melhorias podem estar relacionadas com a interação entre $\mathrm{Nb}_{2} \mathrm{O}_{5}$ e $\mathrm{LiF}$, representada pelas fases $\mathrm{Nb}_{3} \mathrm{O}_{7} \mathrm{~F}$ e $\mathrm{LiNbO}_{3}$.

\section{Agradecimentos}

Os autores gostariam de agradecer à CAPES pelo financiamento. 


\section{REFERÊNCIAS}

1 Aragon-Duarte MC, Nevarez-Rascon A, Esparza-Ponce HE, et al. Nanomechanical properties of zirconia- yttria and alumina zirconia-yttria biomedical ceramics, subjected to low temperature aging. Ceramics International. 2017;43:3931-3939.

2 Guo W-M, Zhang Z-L, Li, J-X, et al. Improvement of densification and mechanical properties or $\mathrm{Al}_{2} \mathrm{O}_{3}-\mathrm{B}_{4} \mathrm{C}$ ceramics. Ceramics International. 2016;42:11486-11489.

3 Maity A, Kayal N, Chakrabarti O. Mechanical behaviour of reaction processed SiC ceramics from artificial precursor from plant. Ceramics International. 2016;42:1005810065.

$4 \mathrm{Li} \mathrm{B}, \mathrm{Xu} \mathrm{Y}$, Zhang S. The size-effect of $\mathrm{Al}_{2} \mathrm{O}_{3}$ on the sinterability, microstructure and properties of glass-alumina composites. Glass Physics and Chemistry. 2015;41:503508.

5 Lu Z, Jiang D, Zhang J, et al. ZrB2-SiC laminated ceramic composites. Journal of the European Ceramic Society. 2012;32:1435-1439.

6 Zhang $\mathrm{X}-\mathrm{Y}, \mathrm{Wu} \mathrm{J}-\mathrm{M}$, Yang J-L. Pore morphology designs of porous $\mathrm{Si}_{3} \mathrm{~N}_{4}$-based ceramics using $\mathrm{Si}_{3} \mathrm{~N}_{4}$ and $\mathrm{Al}_{2} \mathrm{O}_{3}$ poly-hollow microspheres as pore-forming agentes. Materials Letters. 2015;144:39-42

7 Zang W, Guo F, Liu J, et al. Lightweight alumina based fibrous ceramics with different high temperature binder. Ceramics International. 2016;42:10310-10316.

8 Hassan AM, Awaad, M, Bondioli F, Densification Behavior and Mechanical Properties of Niobium-Oxide-Doped Alumina Ceramics. Journal of Ceramic Science and Technology. 2014;5:51-56.

9 Liu $\mathrm{Y}$, Min F, Zhu J, Zhang M. Effect of nanometer $\mathrm{Al}_{2} \mathrm{O}_{3}$ powder on microstructure and properties of alumina ceramics by microwave sintering. Materials Science and Engineering: A. 2012;546:328-331.

10 Zhu B, Zhu Y, Li X, Zhao F. Effect of ceramic bonding phases on the thermomechanical properties of $\mathrm{Al}_{2} \mathrm{O}_{3}-\mathrm{C}$ refractories. Ceramics International. 2013;39:60696076.

11 Nogueira FGE, Ascencios YJO, Rodella CB, Porto ALM, Assaf EM. Alternative route for the synthesis of high surface-area $\eta-\mathrm{Al}_{2} \mathrm{O}_{3} / \mathrm{Nb}_{2} \mathrm{O}_{5}$ catalyst from aluminum waste. Materials Chemistry and Physics. 2016;184:23-30.

12 Oliveira LNL, Campos RVB, Gouveia DX, Silva MAS, Sombra SB. Microwave dielectric properties study of $\left(\mathrm{Al}_{2} \mathrm{O}_{3}\right)-\left(\mathrm{Nb}_{2} \mathrm{O}_{5}\right)$ composite for dielectric resonator antenna applications. Microwave and Optical Technology Letters. 2016;58:1473-1479.

13 Jang JH, Kim TY, Kim NJ, Lee CH, Park EM, Park C, Suh SJ. Preparation and characterization of $\mathrm{Nb}_{2} \mathrm{O}_{5}-\mathrm{Al}_{2} \mathrm{O}_{3}$ composite oxide formed by cathodic electroplating and anodizing. Materials Science and Engineering B. 2011;176:1505-1508.

14 Gomes AV. Comportamento balística da alumina com adição de nióbia e variação da geometria do alvo. Tese de doutorado - IME. Rio de Janeiro. 2004.

15 Trindade W. Influência da Geometria e da Microestrutura no Comportamento Dinâmico da Alumina Adititivada com Nióbia. Tese de doutorado - IME. Rio de Janeiro, 2012.

16 Sutorik AC, Cooper C, Gilde G. Visible light transparency for polycrystalline ceramics of $\mathrm{MgO}-2 \mathrm{Al}_{2} \mathrm{O}_{3}$ and $\mathrm{MgO}-2.5 \mathrm{Al}_{2} \mathrm{O}_{3}$ spinel solid solutions.J. Am. Ceram. Soc. 2013;96:3704-3707.

17 Sutorik AC, Gilde G, Cooper C, Wright J, Hilton C. The effect ot varied amounts of LiF sintering aid on the transparency of alumina rich spinel ceramic with the composition MgO-1.5 $\mathrm{Al}_{2} \mathrm{O}_{3}$. J. Am. Ceram. Soc. 2012;95:1807-1810.

18 Santos JL, Marçal RLSB, Jesus PRR, Gomes AV, Lima Jr EP, Monteiro SN, de Campos JB, Louro LHL Effect of LiF as sintering agent on the densification and phase formation in Al2O3-4wt\%Nb2O5 ceramic compound. Metallurgical and Materials Transactions A. aceito para publicação em 2017.

19 NBR 6220. Material refratáriodenso - determinação da densidade de massaaparente, porosidadeaparente, absorção e densidadeaparente da parte sólida. ABNT. $2^{2}{ }^{a}$ edição; 2011. 
20 Liu $\mathrm{M}$, Xue $\mathrm{D}$, Luo $\mathrm{C}$. Wet chemical synthesis of pure $\mathrm{LiNbO}_{3}$ powders from simple niobium oxide Nb2O5. Journal of Alloys and Compounds. 2006;426:118-122.

21 German Randall M, Suri P, Park SJ. Review: liquid phase. Sintering J. Mater. Sc. 2009;44:1-39. 